\title{
A COMPARISON OF SIMILARITY MEASURES FOR CLUSTERING OF QRS COMPLEXES
}

\author{
Kuang-Chiung Chang ${ }^{1}$, Cheng Wen ${ }^{1}$, Ming-Feng Yeh ${ }^{1}$, Ren-Guey LeE ${ }^{2}$ \\ ${ }^{1}$ Department of Electrical Engineering, Lunghwa University of Science and Technology, \\ Taoyuan, Taiwan \\ ${ }^{2}$ Department of Electronic Engineering, National Taipei University of Technology, Taipei, Taiwan
}

\begin{abstract}
Similarity or distance measures play important role in the performance of algorithms for ECG clustering problems. This paper compares four similarity measures such as the city block $\left(L_{1}\right.$-norm), Euclidean ( $L_{2}$-norm), normalized correlation coefficient, and simplified grey relational grade for clustering of QRS complexes. Performances of the measures include classification accuracy, threshold value selection, noise robustness, execution time, and the capability of automated selection of templates. The clustering algorithm used is the so-called two-step unsupervised method. The best out of the 10 independent runs of the clustering algorithm with randomly selected initial template beat for each run is used to compare the performances of each similarity measure. To investigate the capability of automated selection of templates for ECG classification algorithms, we use the cluster centers generated by the clustering algorithm with various measures as templates. Four sets of templates are obtained, each set for a measure. And the four sets of templates are used in the knearest neighbor classification method to evaluate the performance of the templates. Tested with MIT/BIH arrhythmia data, we observe that the simplified grey relational grade outperforms the other measures in classification accuracy, threshold value selection, noise robustness, and the capability of automated selection of templates.
\end{abstract}

Biomed Eng Appl Basis Comm, 2005(December); 17: 324-331.

Keywords:similarity measure; grey relational grade; ECG clustering; ECG classification

\section{INTRODUCTION}

The electrocardiogram (ECG) is the record of variation of bioelectric potential with respect to time as the human heartbeats. Due to the ease of recording ECG in a noninvasive manner, ECG plays an important role in patient monitoring and diagnosis today. Visual analysis of long term ECG is tedious since a large

Received: August 12, 2005; Accepted: Oct 27, 2005 Correspondence: Kuang-Chiung Chang, Professor Department of Electrical Engineering, Lunghwa University of Science and Technology, Taoyuan, Taiwan E-mail: kcchang@mail.lhu.edu.tw amount of data may be obtained in the case of monitoring ECG signals of a patient continuously during 24 hours. In this case, typically, there could be more then 100 thousands heartbeats generated a day for analysis. Computer-based methods for analysis and interpretation of ECGs have been developed in order to overcome this difficulty. However, classification or clustering of the ECG is a difficult problem even with the aid of a computer, since ECGs differ significantly even for the same type and for the same patient. Due to the importance of the problem, a lot of efforts and methodological studies have been reported on this subject.

Generally speaking, in classification algorithms 
for the ECG, a similarity measure is used to measure the distances between the query beat and the templates in the database, or adjustable weights in the artificial neural network used, for ranking. The smaller the distance is, the more similar the template or the adjustable weight is to the query. Then, a threshold value on the measurements is used for the help with the decision-making. It is known that the distance or similarity measure used in a clustering or classification algorithm will have large effect on the performance of the classification procedure. In [1], performance of several similarity measures in four types of clustering algorithms was investigated on the practical medical data set containing both numerical and categorical of attributes. In [2], nine image similarity measures were compared for texture image retrieval. In this paper, we compare similarity measures for clustering of the QRS complexes.

Distance measures widely used in the time domain ECG classification algorithms include the $\mathrm{L}_{1}$-norm (city block) [4], $\mathrm{L}_{2}$-norm (Euclidean distance) $[3,5]$ normalized cross correlation [6], likelihood function [7], and Mahalanobis distance [8]. In Mahalanobis distance, the covariance matrices can be hard to determine accurately, and the memory and the time requirements grow quadratically rather than linearly with the number of features. These problems may be insignificant when only a few features of the ECG are needed, but they become quite serious when the number of features becomes large. A likelihood function is usually used in an algorithm such as a linear discriminant, where the likelihood function of the data is maximized with respect to the parameters of the model. The model is of suitable functional form with parameters to be adjusted so that it approximates the distribution of the data. The computational load of this approach is very high.

The grey relational grade [9] is also a distance measure whose value is within the interval $(0,1]$. For a given reference sequence and a given set of comparative sequences, grey relational analysis [9] can be used to determine the grey relational grade between the reference and each comparative sequence in the given set. Then the best comparative sequence is the one having the largest grey relational grade. Recently, an unsupervised algorithm has been developed by using a modified grey relational grade as the distance measure for data clustering and showed that it could achieve a good performance [10]. The original grey relational grade is simplified for the reduction of computation, in this paper.

In this paper, we compare performances of four similarity measures, namely, the $\mathrm{L}_{1}$-norm, the $\mathrm{L}_{2}$-norm, the normalized cross correlation, and the simplified grey relational grade, that are relatively simple in computation. Performances of the measures include classification accuracy, threshold value selection, noise robustness, execution time, and the capability of automated selection of templates. The four similarity measures are compared in a clustering algorithm for clustering the ECG. The clustering algorithm used in this paper is the so-called two-step unsupervised method [6], which was reported to have a better performance than the hierarchical cluster analysis approach. It is known that the initial template beat may affect the performance of clustering algorithms. To avoid this, the best out of the 10 independent runs of the clustering algorithm with randomly selected initial template beat for each run is used to compare the performances of each similarity measure. For each run, the threshold values used for the decision-making are selected to make the algorithm have the best result.

The resulting cluster centers of the clustering algorithm can be used as templates representing that cluster. In this viewpoint, the cluster centers may have the potential to be templates that can be used in algorithms for real time classification of the ECG. To investigate the capability of automated selection of templates of the measures for ECG classification algorithms, we use the cluster centers generated by the clustering algorithm with each of the four measures as templates. Four sets of templates are obtained, each set for a measure. And the four sets of templates are used in the k-nearest neighbor classification method to evaluate the performance of the templates.

The remainder of this paper is organized as follows. The simplified grey relational grade is developed in Section 2. Section 3 describes the ECG data and the methods used. Experimental results are shown in Section 4. Finally, some conclusions are given in Section 5.

\section{SIMPLIFIED GREY RELATIONAL GRADE AND THE OTHER MEASURES}

In this section, we introduce the grey relational grade developed in [9] and simplify it for our purpose. Denote $y=\langle y(1), y(2), \ldots y(n)\rangle$ as the reference sequence, and $x_{i}=\left\langle x_{i}(1), x_{i}(2), \ldots x_{i}(n)\right\rangle, i=1,2, \ldots, m$, as the comparative sequences. Define the grey relational coefficient between $y$ and $x_{i}$ at the $k$-th datum as [9]

$$
r\left(y(k), x_{i}(k)\right)=\frac{\Delta_{\min }+\xi \cdot \Delta_{\max }}{\Delta_{i}(k)+\xi \cdot \Delta_{\max }}
$$

where $\Delta_{i}(k)=\left|y(k)-x_{i}(k)\right|, \quad \Delta_{\max }=\max _{i} \max _{k} \Delta_{i}(k)$,
$\Delta_{\min }=\min _{i} \min _{k} \Delta_{i}(k), \quad k=1,2, \ldots, n$, and $\xi \in(0,1]$ is the distinguishing coefficient controlling the resolution between $\Delta \max$ and $\Delta \min$ The weighted average of the 
grey relational coefficients, termed by the grey relational grade, can be computed by

$$
g\left(y, x_{i}\right)=\sum_{k=1}^{n}\left[w(k) \cdot r\left(y(k), x_{i}(k)\right)\right]
$$

where $w(k) \geq 0$ is the weighting function with $\sum_{k=1}^{n} w(k)=1$. The weighting function can be seen as a window that can be used to emphasize a segment of ECG signal such as $\mathrm{P}$ wave. In this paper, we deal only with QRS complex classification problem, and we select the weighting function as $w(k)=1 / n$ for all $k$ From (1a) and (2), it can be seen that $0<g\left(y, x_{i}\right) \leq 1$ and that the smaller the absolute value $\Delta_{i}(k)$, the larger the grey relational grade, and vice versa. The best comparative sequence is the one with the largest grey relational grade to the reference. Hence, the grey relational grade can be used as a distance measure.

The main difficulty of using (1a) is it needs to determine two control factors, $\Delta \max$ and $\Delta \min$. These two control factors may vary as a new reference sequence is presented to a fixed set of comparative sequences. In this case, the analytical process must be done allover again in order to calculate $\Delta \max$ and $\Delta$ min and to obtain the newly best comparative one. It seems inefficient for a real-time ECG analysis. Therefore, we propose an approach in the following for simplifying the computation. From the definition of $\Delta$ min, we observe that if we define a temporary set by adding the reference into original set of comparative sequences then $\Delta \min =0$ for the temporary set of comparative sequences to the reference. By doing this, the best comparative sequence in the temporary set to the reference is always the reference itself. However, it was pointed out that adding a new comparative sequence might change the values of the grey relational grade of original comparative sequences, but it did not change their relative rank [9]. Therefore, for any reference sequence, we can always let $\Delta \min =0$ then the best comparative sequence in the original set is the one having the largest grey relational grade except the reference itself in the temporary set, and it is the same one having the largest grey relational grade in the original set.

Now, the problem of finding the best comparative sequence by using (1a) and (2) is reformulated as finding the best comparative sequence by using (1b) in the below and (2).

$$
r\left(y(k), x_{i}(k)\right)=\frac{\xi \cdot \Delta_{\max }}{\Delta_{i}(k)+\xi \cdot \Delta_{\max }} .
$$

Inspiring of the dependence of the value of $\Delta \max$ to the reference sequence, we replace (1b) with the one in the following.

$$
r\left(y(k), x_{i}(k)\right)=\frac{d_{\max }}{\Delta_{i}(k)+d_{\max }}
$$

where $d_{\max }$ is a positive real constant. If we use (1c) and (2) as the similarity measure for ranking the comparative sequences, the result will be the same as that obtained by using (1b) and (2). By this way, the best comparative sequence can be found by using (1c) and (2). The grade obtained by (1c) and (2) is called the simplified grey relational grade, denoted by $d_{s g}$, in this paper. The range of the simplified grey relational grade is still in the interval $(0,1)$. Note that, for a specific sequence in the comparative set, its simplified grey relational grade value and its grey relational grade value obtained by (1a) and (2) may be different, but the rank of the sequence in that set is always the same by using either of the three measures.

The other measures used in this paper are summarized in the following. Denote the city block or $\mathrm{L}_{1}$ distance of two sequences $x$ and $y$ by

$$
d_{L 1}(y, x)=\sum_{k=1}^{n}|y(k)-x(k)| .
$$

The Euclidean distance or L2 distance is defined by

$$
d_{L 2}(y, x)=\sqrt{\sum_{k=1}^{n}(y(k)-x(k))^{2}} .
$$

The Euclidean distance is not always the best measure. The fact that the distances in each element of the sequences are squared before summation places great emphasis on those elements for which the dissimilarity is large. Finally, the normalized cross correlation is defined by

$$
d_{c c}(y, x)=\frac{\sum_{k=1}^{n}(y(k)-\bar{y})(x(k)-\bar{x})}{\sqrt{\sum_{k=1}^{n}(y(k)-\bar{y})^{2}(x(k)-\bar{x})^{2}}}
$$

where

$$
\bar{y}=\frac{1}{n} \sum_{k=1}^{n} y(k) \quad \bar{x}=\frac{1}{n} \sum_{k=1}^{n} x(k)
$$

A distance function with a wide range may cause a problem in the selection of the threshold value for decision-making when it is used in a clustering algorithm. From the definition of each distance function, we see that $d_{L 1} \in[0, \infty), d_{L 2} \in[0, \infty), d_{c c} \in[-1,1]$, 
and $d_{s g} \in(0,1]$. For the easy selection of the threshold value, $d_{L 1}$ and $d_{L 2}$ are usually normalized, and the absolute value of $d_{c c}$ is used in clustering algorithms. Let the normalized $d_{L 1}$ and $d_{L 2}$ be denoted by $\hat{d}_{L 1}$ and $\hat{d}_{L 2}$, respectively. Finally, define the similarity measure for each distance function as

$$
\begin{aligned}
& s_{L 1}=1-\hat{d}_{L 1} \\
& s_{L 2}=1-\hat{d}_{L 2} \\
& s_{c c}=\left|d_{c c}\right| \\
& s_{s g}=d_{s g} .
\end{aligned}
$$

From these definitions, we see that all similarity measures lay within the range $[0,1]$ except $s_{s g} \in(0,1]$ and that, for all similarity measures, the larger the measurement of two sequences, the more similar of the two sequences.

\section{MATERIAL AND METHOD}

The MIT/BIH Arrhythmia Database [11], sampled at $360 \mathrm{~Hz}$ with 11-bit resolution, was used in this study. Excluding records containing paced beats (102, 104,107 , and 217), we used the other 44 records of the MIT/BIH Database. Raw data from single channel (channel 1) were used (with some beats missing). The size of each QRS complex was 73 samples (about 200 $\mathrm{ms}$ ) with the $\mathrm{R}$ wave at the center. This study did not investigate the problem of QRS detection from the ECG, instead the position of annotation labels provided with the MIT/BIH Database was used to identity the $\mathrm{R}$ wave. The AAMI recommended practice [12] was used to combine the MIT/BIH beat types into four classes. Class $\mathrm{N}$ contains normal, bundle branch block, and supraventricular ectopic beats, class V contains ventricular ectopic beats, class $\mathrm{F}$ contains fusion of ventricular and normal beats, and class $Q$ contains unclassifiable beats. The detail is listed in Table I.

Without feature extraction, the QRS morphology with 73 samples was used in this study. To test the robustness of the similarity measures to high frequency noise and power line interference, we did not use any data-preprocessing step such as filtering in this study. However, the mean value was subtracted from each beat to remove the baseline wander [3].

The so-called two-step clustering algorithm used in this study was suggested in [6]. The algorithm takes the first beat of the ECG as the template and compares it to all successive beats by one of the similarity measures (6)-(9). The beats showing certain degree of similarity are put together to form a cluster. By this we mean that if the measurement exceeds a threshold value, the compared beat belongs to the same cluster as the template. If no measurement exceeds the threshold value, the template itself forms a cluster. When all successive beats have been compared, the first beat of the residual QRS complexes is taken as a new template, and it is used to iterate the whole procedure until no QRS complex is left unclassified. In the second step of the algorithm, the clusters are merged by the same way used in the first step. However, in this step, we use the cluster center to represent the cluster and select a slightly lower threshold value than that used in step one. Finally, all beats in one-element clusters are collected in a signal cluster, and they are assigned as unclassifiable beats (class Q).

The two-step clustering algorithm described in the above was used to evaluate performances of the four similarity measures (6)-(9). For a fair comparison, we used the best result of each measure for comparing the performances of each measure. As mentioned before, the performances include the accuracy, the execution time, the variation of threshold for the best result for each record, and the noise robustness. In addition, we evaluated the capability of the measures for the automated selection of templates from an existing ECG recording of a specific patient for designing a customized ECG classifier for that patient [3]. The two-step clustering algorithm is designed for analyzing existing long term ECG recordings that may be obtained by a Holter type monitor, and it is not for real time analysis. When real time analysis is needed, we may use the clustering algorithm to analyze the previous recorded ECG of the patient, select templates from each resulting cluster automatically, recognize the templates by an expert, and use the templates in some classification algorithms for real time analysis such as the $k$-nearest neighbors method described in the below. This approach could be used to design a customized real time ECG classifier. By this way, only a few

Table I . Mapping the MIT/BIH database beat types to the AAMI beat classes

\begin{tabular}{lcc}
\hline \multicolumn{1}{c}{ Description } & MIT label & Test label \\
\hline Normal beat & 1 & $\mathrm{~N}$ \\
Left bundle branch block beat & 2 & $\mathrm{~N}$ \\
Right bundle branch block beat & 3 & $\mathrm{~N}$ \\
Bundle branch block beat (unspecified) & 25 & $\mathrm{~N}$ \\
Nodal (junctional) premature beat & 7 & $\mathrm{~N}$ \\
Atrial premature beat & 8 & $\mathrm{~N}$ \\
Supraventricular premature beat & 9 & $\mathrm{~N}$ \\
Abberrated atrial premature beat & 4 & $\mathrm{~N}$ \\
Nodal (junctional) escape beat & 11 & $\mathrm{~N}$ \\
Atrial escape beat & 34 & $\mathrm{~N}$ \\
Ventricular premature beat & 5 & $\mathrm{~V}$ \\
R-on-T ventricular premature beat & 41 & $\mathrm{~V}$ \\
Ventricular escape beat & 10 & $\mathrm{~V}$ \\
Fusion of ventricular and normal beat & 6 & $\mathrm{~F}$ \\
Unclassifiable beat & 13 & $\mathrm{Q}$ \\
Others & $\mathrm{Other}$ & $\mathrm{Q}$ \\
\hline
\end{tabular}


templates need to be recognized manually. Instead of the resulting cluster center, the ECG beat nearest to the cluster center was selected as the template. For simplicity, only the measure with the best performance in the two-step algorithm was used in the classification algorithm.

The customized ECG classifier was implemented based on the k-nearest neighbors method. In the $k$ nearest neighbors algorithm, by the measure used, the query is compared to templates representing classes or types of the QRS (the class Q had no templates for it). Then, the similarity measurements are ranked, and the largest measurement exceeding a pre-defined threshold value indicates to which class the query belongs. When no measurement exceeds the threshold value, class $Q$ is assigned to the query.

\section{EXPERIMENAL RESULT}

All programs coded by Matlab ${ }^{\circledR}$ version R12 were executed by a personal computer with Intel ${ }^{\circledR}$ Pentium ${ }^{\circledR}$ $41.6 \mathrm{GHz}$ processor and $512 \mathrm{MB}$ RAM. In the twostep clustering algorithm, different initial templates may result in different performances. To have a fair comparison, we randomly selected 10 ECG beats from each test record as the initial templates for that record. Then, for each record, we used 10 runs, one initial template for a run, to evaluate the performances of each of the 4 similarity measures. By this way, each similarity measure was evaluated 10 times independently under the same clustering method with the same initial template for every test record. Then, for each measure, the best result of the 10 runs was selected for the comparison. In each run, the threshold value used in the first step of the algorithm was selected to make the algorithm have the best performance in accuracy, and in the second step it was selected to minimize the resulting number of clusters while keeping the accuracy obtained by the first step.

In respect to the performance in accuracy, the best clustering result of such a 10 runs for each measure for each of the selected MIT/BIH ECG record is listed in Table II. Table II shows that, over total 101,374 test ECG beats, $S_{L 1}, S_{L 2}, S_{c c}$, and $S_{s g}$ achieve $99.46 \%$, $99.38 \%, 99.66 \%$, and $99.68 \%$ in accuracy, respectively. The simplified grey relational grade outperforms the other similarity measure functions in classification accuracy. Table III contains the details of the best result obtained by using $S_{s g}$ for each record.

Table II also shows that the performance in accuracy of each measure on noisy records. Over 62,710 beats in 27 noisy records, $S_{L 1}, S_{L 2}, S_{c c}$, and $S_{s g}$ achieve $99.50 \%, 99.46 \%, 99.82 \%$, and $99.83 \%$ in accuracy, respectively. The simplified grey relational grade has the best performance in accuracy on noisy records. It seems that all measures perform better on noisy records than clean records. This is because all of the worst cases of the measures appear on record 213 that is labeled clean. Excluding record 213, all measures perform better on clean records as shown in Table II.

Fig. 1 shows the variation of the threshold value used in the first step of the algorithm. Note that the threshold value reported is the minimum value for achieving the best performance in accuracy. For values larger than the minimum value, the accuracy can also be achieved but with a larger number of resulting clusters in this step. Though the resulting clusters in the first step will be merged in the second step of the algorithm, a larger number of clusters cause a more computational load in the second step. This figure depicts that, for different records, the minimal threshold value required for the simplified grey relational grade achieving the best result is relatively stable, comparing with the other measures. This is a particularly useful feature since we need not spend much time to decide the threshold, in practice, for analyzing various ECG recordings. The threshold in the second step of the algorithm is used to minimize the number of clusters by merging them. No significant difference in the number of final resulting clusters was observed for different measures. Once, the threshold values for the first and the second steps were decided for every record, we ran the program again with the fixed threshold values to examine the execution time for each measure. The result is also listed in Table $\Pi$. From Table $\Pi$, it is seen that, over 101,374 test beats, the clustering algorithm with the $\mathrm{L}_{1}$ function takes the least time $(35.73 \mathrm{sec}$.). The computational load caused by the additional procedure for normalizing the $\mathrm{L}_{1}$ or the $\mathrm{L}_{2}$ function seems that it can be negligible.

In a final cluster obtained by the clustering algorithm, the ECG beat being nearest to the cluster center was selected as the template for the $k$-nearest neighbors classification method to investigate the

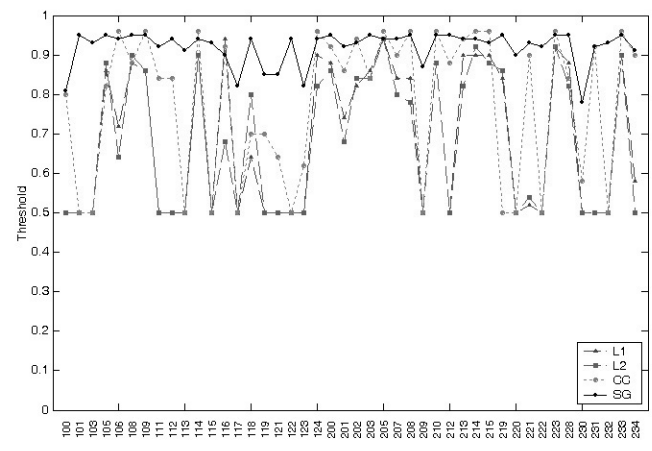

Fig 1. The variation of the thresholds used in the first step of the clustering algorithm. 
Table II . Results of ECG beats clustering using various measures on 44 MIT/BIH ECG data files

\begin{tabular}{|c|c|c|c|c|c|c|}
\hline \multirow{2}{*}{ Record } & \multirow{2}{*}{ No. of beats ${ }^{-}$} & \multicolumn{4}{|c|}{ Accuracy (\%) } & \multirow{2}{*}{ Noisy } \\
\hline & & $S_{L 1}$ & $s_{L 2}$ & $S_{c c}$ & $S_{s g}$ & \\
\hline 100 & 2271 & 100 & 100 & 100 & 100 & \\
\hline 101 & 1864 & 100 & 100 & 100 & 100 & $\mathrm{Y}$ \\
\hline 103 & 2084 & 100 & 100 & 100 & 100 & $\mathrm{Y}$ \\
\hline 105 & 2572 & 98.60 & 99.61 & 99.96 & 99.73 & $\mathrm{Y}$ \\
\hline 106 & 2027 & 98.67 & 96.35 & 100 & 99.95 & \\
\hline 108 & 1773 & 99.38 & 99.89 & 99.94 & 99.89 & $\mathrm{Y}$ \\
\hline 109 & 2531 & 100 & 99.96 & 100 & 100 & \\
\hline 111 & 2124 & 100 & 100 & 100 & 100 & $\mathrm{Y}$ \\
\hline 112 & 2539 & 100 & 100 & 100 & 100 & $\mathrm{Y}$ \\
\hline 113 & 1794 & 100 & 100 & 100 & 100 & \\
\hline 114 & 1879 & 100 & 99.89 & 99.89 & 99.84 & $\mathrm{Y}$ \\
\hline 115 & 1952 & 100 & 100 & 100 & 100 & $\mathrm{Y}$ \\
\hline 116 & 2411 & 99.96 & 99.96 & 100 & 99.96 & \\
\hline 117 & 1534 & 100 & 100 & 100 & 100 & $\mathrm{Y}$ \\
\hline 118 & 2287 & 99.78 & 99.96 & 99.96 & 99.96 & $\mathrm{Y}$ \\
\hline 119 & 1987 & 100 & 100 & 100 & 100 & \\
\hline 121 & 1863 & 100 & 100 & 100 & 100 & Y \\
\hline 122 & 2476 & 100 & 100 & 100 & 100 & \\
\hline 123 & 1517 & 100 & 100 & 100 & 100 & \\
\hline 124 & 1619 & 99.94 & 99.82 & 100 & 100 & \\
\hline 200 & 2600 & $\begin{array}{l}99.69 \\
\end{array}$ & 99.35 & 99.92 & 99.92 & $\bar{Y}$ \\
\hline 201 & 2000 & 99.55 & 99.50 & 100 & 100 & Y \\
\hline 202 & 2136 & 100 & 100 & 100 & 100 & \\
\hline 203 & 2980 & 97.68 & 98.05 & 99.66 & 99.66 & $\mathrm{Y}$ \\
\hline 205 & 2656 & 99.74 & 99.81 & 99.70 & 99.74 & \\
\hline 207 & 2331 & 97.43 & 94.21 & 99.96 & 99.96 & $\mathrm{Y}$ \\
\hline 208 & 2953 & 97.97 & 97.56 & 97.53 & 98.04 & $\mathrm{Y}$ \\
\hline 209 & 3004 & 100 & 100 & 100 & 100 & \\
\hline 210 & 2650 & 99.58 & 99.77 & 99.93 & 99.55 & Y \\
\hline 212 & 2747 & 100 & 100 & 100 & 100 & $\mathrm{Y}$ \\
\hline 213 & 3250 & 95.94 & 95.54 & 95.02 & 95.54 & \\
\hline 214 & 2260 & 98.85 & 99.87 & 99.74 & 99.87 & $\mathrm{Y}$ \\
\hline 215 & 3363 & 100 & 100 & 100 & 100 & $\mathrm{Y}$ \\
\hline 219 & 2287 & 97.60 & 97.60 & 97.51 & 97.55 & \\
\hline 220 & 2047 & 100 & 100 & 100 & 100 & \\
\hline 221 & 2427 & 99.67 & 99.51 & 100 & 100 & $\mathrm{Y}$ \\
\hline 222 & 2482 & 100 & 100 & 100 & 100 & $\mathrm{Y}$ \\
\hline 223 & 2605 & 99.54 & 99.62 & 99.54 & 99.62 & $\mathrm{Y}$ \\
\hline 228 & 2053 & 99.95 & 99.95 & 100 & 100 & $\mathrm{Y}$ \\
\hline 230 & 2255 & 100 & 100 & 100 & 100 & Y \\
\hline 231 & 1573 & 99.94 & 99.94 & 100 & 100 & \\
\hline 232 & 1780 & 100 & 100 & 100 & 100 & $\mathrm{Y}$ \\
\hline 233 & 3078 & 99.77 & 99.81 & 99.84 & 99.84 & \\
\hline 234 & 2753 & 100 & 100 & 100 & 100 & Y \\
\hline Total & 101374 & 99.46 & 99.38 & 99.66 & 99.68 & \\
\hline $\begin{array}{l}\text { Beats in noisy } \\
\text { records }\end{array}$ & 62710 & 99.50 & 99.46 & 99.82 & 99.83 & \\
\hline $\begin{array}{l}\text { Beats in clean } \\
\text { records }\end{array}$ & 38664 & 99.40 & 99.25 & 99.40 & 99.44 & \\
\hline $\begin{array}{l}\text { Beats in clean } \\
\text { records } \\
\text { excluding } 213 \\
\end{array}$ & 35415 & 99.72 & 99.59 & 99.80 & 99.80 & \\
\hline $\begin{array}{l}\text { Execution } \\
\text { time (sec) }\end{array}$ & & 35.73 & 38.06 & 50.05 & 41.50 & \\
\hline
\end{tabular}

capability of the measures for the automated selection of templates. For each record, there were four sets of templates, each for a measure. For simplicity, only the set of templates generated by using the simplified grey relational grade is reported in Table IV. Note that there are no templates for class $\mathrm{Q}$ in Table IV.

The measurement of the $\mathrm{L}_{1}$ or the $\mathrm{L}_{2}$ distance function needs to be normalized over all measurements before comparing with the threshold for assigning the query to class Q. Therefore, the two distance functions are inadequately to be measures in real time classification algorithms. On the other hand, the normalized correlation coefficient function performed
Table III . Details of the clustering result using the simplified gray relation grade

\begin{tabular}{|c|c|c|c|c|c|c|c|c|c|c|}
\hline \multirow{2}{*}{ Record- } & \multicolumn{5}{|c|}{ Number of beats } & \multicolumn{5}{|c|}{ Clustering results (true positive) } \\
\hline & $\mathrm{N}$ & $\mathrm{V}$ & $\mathrm{F}$ & $\mathrm{Q}$ & Sum & $\mathrm{N}$ & $\mathrm{V}$ & $\mathrm{F}$ & $\mathrm{Q}$ & AcC $(\%)$ \\
\hline 100 & 2270 & 1 & & & 2271 & 2270 & 1 & & & 100 \\
\hline 101 & 1862 & & & 2 & 1864 & 1862 & & & 2 & 100 \\
\hline 103 & 2084 & & & & 2084 & 2084 & & & & 100 \\
\hline 105 & 2526 & 41 & & 5 & 2572 & 2523 & 40 & & 2 & 99.73 \\
\hline 106 & 1507 & 520 & & & 2027 & 1507 & 519 & & & 99.95 \\
\hline 108 & 1744 & 16 & 2 & 11 & 1773 & 1744 & 16 & 1 & 10 & 99.49 \\
\hline 109 & 2491 & 38 & 2 & & 2531 & 2491 & 38 & 2 & & 100 \\
\hline 111 & 2123 & 1 & & & 2124 & 2123 & 1 & & & 100 \\
\hline 112 & 2539 & & & & 2539 & 2539 & & & & 100 \\
\hline 113 & 1794 & & & & 1794 & 1794 & & & & 100 \\
\hline 114 & 1832 & 43 & 4 & & 1879 & 1832 & 43 & 1 & & 99.84 \\
\hline 115 & 1952 & & & & 1952 & 1952 & & & & 100 \\
\hline 116 & 2302 & 109 & & & 2411 & 2302 & 108 & & & 99.96 \\
\hline 117 & 1534 & & & & 1534 & 1534 & & & & 100 \\
\hline 118 & 2261 & 16 & & 10 & 2287 & 2261 & 16 & & 9 & 99.96 \\
\hline 119 & 1543 & 444 & & & 1987 & 1543 & 444 & & & 100 \\
\hline 121 & 1862 & 1 & & & 1863 & 1862 & 1 & & & 100 \\
\hline 122 & 2476 & & & & 2476 & 2476 & & & & 100 \\
\hline 123 & 1514 & 3 & & & 1517 & 1514 & 3 & & & 100 \\
\hline 124 & 1567 & 47 & 5 & & 1619 & 1567 & 47 & 5 & & 99.81 \\
\hline 200 & 1772 & 826 & 2 & & 2600 & 1772 & 825 & 1 & & 99.19 \\
\hline 201 & 1763 & 198 & 2 & 37 & 2000 & 1763 & 198 & 2 & 37 & 99.7 \\
\hline 202 & 2116 & 19 & 1 & & 2136 & 2116 & 19 & 1 & & 99.95 \\
\hline 203 & 2531 & 444 & 1 & 4 & 2980 & 2531 & 435 & 1 & 3 & 98.59 \\
\hline 205 & 2574 & 71 & 11 & & 2656 & 2574 & 71 & 4 & & 99.74 \\
\hline 207 & 1649 & 682 & & & 2331 & 1649 & 681 & & & 99.28 \\
\hline 208 & 1587 & 992 & 372 & 2 & 2953 & 1577 & 970 & 347 & 1 & 98.04 \\
\hline 209 & 3003 & 1 & & & 3004 & 3004 & 1 & & & 100 \\
\hline 210 & 2445 & 195 & 10 & & 2650 & 2443 & 193 & 2 & & 99.55 \\
\hline 212 & 2747 & & & & 2747 & 2747 & & & & 100 \\
\hline 213 & 2668 & 220 & 362 & & 3250 & 2665 & 198 & 242 & & 95.54 \\
\hline 214 & 2001 & 256 & 1 & 2 & 2260 & 2001 & 253 & & & 99.73 \\
\hline 215 & 3198 & 164 & 1 & & 3363 & 3196 & 164 & 1 & & 99.85 \\
\hline 219 & 2089 & 64 & 1 & 133 & 2287 & 2089 & 62 & 1 & 79 & 97.55 \\
\hline 220 & 2047 & & & & 2047 & 2047 & & & & 100 \\
\hline 221 & 2031 & 396 & & & 2427 & 2031 & 396 & & & 100 \\
\hline 222 & 2482 & & & & 2482 & 2482 & & & & 100 \\
\hline 223 & 2118 & 473 & 14 & & 2605 & 2115 & 453 & 1 & & 98.62 \\
\hline 228 & 1691 & 362 & & & 2053 & 1691 & 360 & & & 99.90 \\
\hline 230 & 2254 & 1 & & & 2255 & 2254 & 1 & & & 100 \\
\hline 231 & 1569 & 2 & & 2 & 1573 & 1569 & 2 & & 2 & 100 \\
\hline 232 & 1780 & & & & 1780 & 1780 & & & & 100 \\
\hline 233 & 2236 & 831 & 11 & & 3078 & 2236 & 831 & 6 & & 99.68 \\
\hline 234 & 2750 & 3 & & & 2753 & 2750 & 3 & & & 100 \\
\hline
\end{tabular}

Table IV. Templates for ECG beats classification

\begin{tabular}{|c|c|c|c|c|c|}
\hline Record & No. of templates & Record & No. of templates & Record & No. of templates \\
\hline 100 & $1 \mathrm{~N} 1 \mathrm{~V}$ & 119 & $1 \mathrm{~N} 2 \mathrm{~V}$ & 213 & 4N6V5F \\
\hline 101 & $1 \mathrm{~N}$ & 121 & $2 \mathrm{~N} 1 \mathrm{~V}$ & 214 & $3 \mathrm{~N} 9 \mathrm{~V}$ \\
\hline 103 & $2 \mathrm{~N}$ & 122 & $4 \mathrm{~N}$ & 215 & $4 \mathrm{~N} 5 \mathrm{~V}$ \\
\hline 105 & $18 \mathrm{~N} 4 \mathrm{~V}$ & 123 & $1 \mathrm{~N} 1 \mathrm{~V}$ & 219 & $7 \mathrm{~N} 5 \mathrm{~V}$ \\
\hline 106 & $2 \mathrm{~N} 9 \mathrm{~V}$ & 124 & 2N6V2F & 220 & $2 \mathrm{~N}$ \\
\hline 108 & $17 \mathrm{~N} 10 \mathrm{~V} 1 \mathrm{~F}$ & 200 & 4N18V1F & 221 & $2 \mathrm{~N} 5 \mathrm{~V}$ \\
\hline 109 & 6N4V1F & 201 & 6N4V2F & 222 & $2 \mathrm{~N}$ \\
\hline 111 & 1N1V & 202 & 10N3V1F & 223 & 6N17V1F \\
\hline 112 & $3 N$ & 203 & 19N20V1F & 228 & $5 \mathrm{~N} 6 \mathrm{~V}$ \\
\hline 113 & $2 \mathrm{~N}$ & 205 & 2N4V2F & 230 & $1 \mathrm{~N} 1 \mathrm{~V}$ \\
\hline 114 & 3N3V1F & 207 & $8 \mathrm{~N} 24 \mathrm{~V}$ & 231 & $1 \mathrm{~N} 2 \mathrm{~V}$ \\
\hline 115 & $1 \mathrm{~N}$ & 208 & 11N13V8F & 232 & $3 \mathrm{~N}$ \\
\hline 116 & $4 \mathrm{~N} 2 \mathrm{~V}$ & 209 & $2 \mathrm{~N} 1 \mathrm{~V}$ & 233 & 4N13V3F \\
\hline 117 & $3 N$ & 210 & 7N14V3F & 234 & 3N1V \\
\hline 118 & $6 \mathrm{~N} 4 \mathrm{~V}$ & 212 & $3 \mathrm{~N}$ & & \\
\hline
\end{tabular}

worse than the simplified grey relational grade in accuracy, the selection of thresholds, noise robustness, and the execution time. As a consequence, the only similarity measure used in the $k$-nearest neighbors classification algorithm was the simplified grey relational grade. The threshold value used for the 
classification was 0.85 for all test records.

Table $\mathrm{V}$ contains the classification results of the $k$-nearest neighbors method with the four sets of templates. Over total 101,374 test ECG beats, the sets of templates generated by using $S_{L 1}, S_{L 2}, S_{c c}$, and $S_{s g}$ in the clustering algorithm achieve $99.53 \%, 99.53 \%$, $99.40 \%$, and $99.61 \%$ in classification accuracy, respectively. The set of templates generated by using the simplified grey relational grade achieves the best classification accuracy.

\section{CONCLUSION}

In this paper, the performances of four similarity measures used in the so-called two-step clustering algorithm for ECG complexes have been investigated. The performances included the classification accuracy, the selection of thresholds, the execution time, noise

Table V . Results of ECG beats classification using various sets of templates

\begin{tabular}{rrrrrr}
\hline \multirow{2}{*}{ Record } & \multirow{2}{*}{ No. of beats } & \multicolumn{5}{c}{ Accuracy $(\%)$} \\
\cline { 3 - 6 } & & $\mathrm{T}_{\mathrm{L} 1}$ & $\mathrm{~T}_{\mathrm{L} 2}$ & \multicolumn{1}{c}{$\mathrm{T}_{\mathrm{CC}}$} & \multicolumn{1}{c}{$\mathrm{T}_{\mathrm{SG}}$} \\
\hline 100 & 2271 & 100 & 100 & 100 & 100 \\
101 & 1864 & 99.95 & 99.95 & 99.95 & 99.95 \\
103 & 2084 & 100 & 100 & 99.95 & 100 \\
105 & 2572 & 99.73 & 99.77 & 99.69 & 99.77 \\
106 & 2027 & 100 & 100 & 100 & 100 \\
108 & 1773 & 99.15 & 99.38 & 98.87 & 99.32 \\
109 & 2531 & 100 & 100 & 100 & 100 \\
111 & 2124 & 100 & 100 & 100 & 100 \\
112 & 2539 & 100 & 100 & 100 & 100 \\
113 & 1794 & 100 & 100 & 100 & 100 \\
114 & 1879 & 100 & 100 & 99.89 & 99.89 \\
115 & 1952 & 100 & 100 & 100 & 100 \\
116 & 2411 & 99.96 & 99.96 & 99.96 & 99.96 \\
117 & 1534 & 100 & 100 & 100 & 100 \\
118 & 2287 & 99.56 & 99.61 & 99.56 & 99.96 \\
119 & 1987 & 100 & 100 & 100 & 100 \\
121 & 1863 & 100 & 100 & 100 & 100 \\
122 & 2476 & 100 & 100 & 100 & 100 \\
123 & 1517 & 100 & 100 & 100 & 100 \\
124 & 1619 & 99.94 & 99.94 & 99.88 & 99.81 \\
\hline 200 & 2600 & 99.77 & 99.73 & 99.73 & 99.65 \\
201 & 2000 & 98.15 & 98.15 & 98.15 & 99.95 \\
202 & 2136 & 100 & 100 & 100 & 100 \\
203 & 2980 & 98.49 & 98.49 & 98.42 & 98.69 \\
205 & 2656 & 99.77 & 99.81 & 99.74 & 99.74 \\
207 & 2331 & 99.40 & 99.40 & 98.07 & 99.36 \\
208 & 2953 & 98.34 & 98.07 & 97.93 & 98.17 \\
209 & 3004 & 100 & 100 & 100 & 100 \\
210 & 2650 & 99.62 & 99.77 & 99.81 & 99.55 \\
212 & 2747 & 100 & 100 & 100 & 100 \\
213 & 3250 & 96.43 & 96.40 & 96.31 & 96.15 \\
214 & 2260 & 99.78 & 99.87 & 99.69 & 99.65 \\
215 & 3363 & 100 & 100 & 99.97 & 99.94 \\
219 & 2287 & 94.18 & 94.18 & 94.62 & 97.55 \\
220 & 2047 & 100 & 100 & 100 & 100 \\
221 & 2427 & 100 & 100 & 100 & 100 \\
222 & 2482 & 100 & 100 & 100 & 100 \\
223 & 2605 & 99.54 & 99.54 & 96.93 & 99.04 \\
228 & 2053 & 99.95 & 99.95 & 99.81 & 99.90 \\
230 & 2255 & 100 & 100 & 100 & 100 \\
231 & 1573 & 99.87 & 99.87 & 100 & 100 \\
232 & 1780 & 100 & 100 & 100 & 100 \\
233 & 3078 & 99.81 & 99.84 & 99.64 & 99.68 \\
234 & 2753 & 100 & 100 & 100 & 100 \\
\hline \hline Total & 101374 & 99.53 & 99.53 & 99.40 & 99.61 \\
\hline & & & & &
\end{tabular}

robustness, and the capability of the automated selection of templates. It is found that the simplified grey relational grade outperforms the other measures in classification accuracy, noise robustness, the selection of thresholds, and the capability of the automated selection of templates. And the best measure in the execution time is the $\mathrm{L}_{1}$ distance function.

\section{ACKNOWLEDGMENT}

This work was supported by the National Science Council, Taiwan, under Grant NSC 94-2213-E-027012.

\section{REFERENCE}

1. Hirano S, Sun X, and Tsumoto S: On similarity measures for cluster analysis in clinical laboratory examination database, in Proc. 2002 IEEE 26th Ann. Int. Computer Software and Applications Conf. 2002; 1170-1175.

2. Kokare M, Chatterji BN, and Biswas PK: Comparison of similarity metrics for texture image retrieval, in Proc. 2003 IEEE Conf. on Convergent Technologies for Asia-Pacific Region 2002; 2: 1517.

3. $\mathrm{Hu} \mathrm{YH}$, Palreddy S, and Tompkins WJ: A patientadaptable ECG beat classifier using a mixture of experts approach, IEEE Trans. Biomed. Eng. 1997; 44: 891-900.

4. Rappaport SH, Gillick L, Moody G.B, and Mark RG: QRS morphology classification: Quantitative evaluation of different strategies, Computers in Cardiology 1982; 33-36.

5. Lagerholm M., Peterson C, Braccini G, Edenbrandt L, and Sornmo L: Clustering ECG complexes using Hermite functions and self-organizing maps, IEEE Trans. Biomed. Eng. 2000; 47: 838-848.

6. Maier $\mathrm{C}$, Dickhaus $\mathrm{H}$, and Gittinger $\mathrm{J}$ : Unsupervised morphological classification of QRS complexes, Computers in Cardiology 1999; 683686.

7. De Chazzal P, O' dwyer M, and Reilly RB: Automatic classification of heartbeats using ECG morphology and heartbeat interval features, IEEE Trans. Biomed. Eng. 2004; 51: 1196-1206.

8. Moraes JCTB, Seixas MO, Vilani FN, and Costa EV: A real time QRS complex classification method using Mahalanobis distance, Computers in Cardiology; 2002: 201-204.

9. Deng JL: Introduction to grey system theory, J. Grey Syst. 1989; 1: 1-24.

10. Chank KC. and Yeh MF: Grey relational analysis 
based approach for data clustering, IEE Proc. Vis. Image Signal Process. 2005; 152: 165-172.

11. MIT/BIH database distributor, Beth Israel Hospital, Biomedical Engineering, Division available in the address: http://ecg.mit.edu/, USA, 1979.

12. Recommended Practice for Testing and Reporting Performance Results of Ventricular Arrhythmia Detection Algorithms, Association for the Advancement of Medical Instrumentation, 1987. 